\title{
Analysis of Location Ambiguity, GDOP and Station Layout of Three- station in Multilateration
}

\author{
Zi Li Xu${ }^{1}$, Dong Lin $\mathrm{He}^{1}$, Jing $\mathrm{Li}^{1, \mathrm{a}}$, Yong Tang ${ }^{1}$ and Jian Hong Yang ${ }^{1}$ \\ ${ }^{1} R \& D$ Center, the $2 n d$ Research Institute of Civil Aviation Administration of China(CAAC), Chengdu, Sichuan Province, China
}

\begin{abstract}
In practice, the two-dimensional TDOA source location in the case of three stations would encounter the ambiguity problem of dual solutions. The relationship between distributions of location curve intersections, GDOP and receiving station layout is numerically analyzed, namely the distributions of location curve intersections and GDOP are determined by the receiving station layout, and the areas of two curve intersections are consistent with these of larger GDOP. These analysis results could serve as reference for avoiding or dealing with the ambiguity problem when multilateration is in application.
\end{abstract}

\section{Introduction}

When the multilateration system is applied to actual target positioning, it will inevitably cause that three receiving stations are only used for two-dimensional target positioning no matter in consideration of costeffectiveness or actual environmental limitation. Even for three-station positioning of three-dimensional target not complying with the positioning principle, as target barometric altitude can often be obtained in practice[1,2], three-dimensional positioning equation can be reduced to two-dimensional positioning equation to realize accurate positioning of target[3], therefore, three-station target positioning has a important place in actual application of multilateration technology.

In hyperbolic multilateration system, target position is obtained through the intersection of hyperbolic curve determined by solving positioning equation. Under the situation of only three receiving stations receiving the target signal, the positioning curve intersection situation is also different due to different relative location between the target and the receiving stations, namely, the positioning curves may generate a point of intersection and also may generate two points of intersection. If the positioning curves generate two points of intersection, it is very difficult to judge which point of intersection is the target's actual position without any prior verification information of the target, in other words, ambiguity appears in solving the target position. However, through analysis on the relationship between three-station positioning curve intersection point distribution, GDOP distribution and stations layout, three-station positioning ambiguity problem can be effectively avoided and target positioning accuracy can be improved by reasonably selecting stations layout.

\section{Numerical simulation analysis of three-station positioning curves intersections point distribution}

For multilateration three-station positioning twodimensional target, the target position can be obtained by the point of intersection determined by the positioning equation

$$
\begin{gathered}
\sqrt{\left(x_{i}-x\right)^{2}+\left(y_{i}-y\right)^{2}}-\sqrt{\left(x_{j}-x\right)^{2}+\left(y_{j}-y\right)^{2}}=c \cdot T D O A_{i j}, \\
i, j=1,2,3, i \neq j
\end{gathered}
$$

Where, $(x, y)$ is position coordinates of the target to be positioned, $\left(x_{i}, y_{i}\right)$ is position coordinates of the receiving stations, $T D O A_{i j}$ is time difference of arrival (TDOA) between the target signal reaching station $i$ and station $j, c$ is signal velocity. Equation (1) determines two independent positioning equations, corresponding to two independent positioning hyperbolic curves. Besides it may result in no point of intersection between two positioning curves due to too large measurement error of Time Difference of Arrival (TDOA) of the signal in practice, with different relative position between the target and the receiving stations, the positioning curve may generate one point of intersection, and also may generate two points of intersection. Two intersection situations of the pointing curves are as shown in Fig. 1.

\footnotetext{
a Corresponding author: jfm1113@163.com
} 


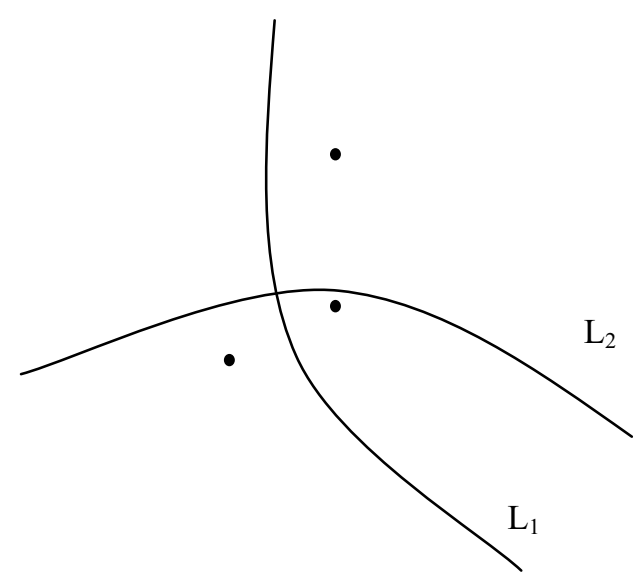

(a)

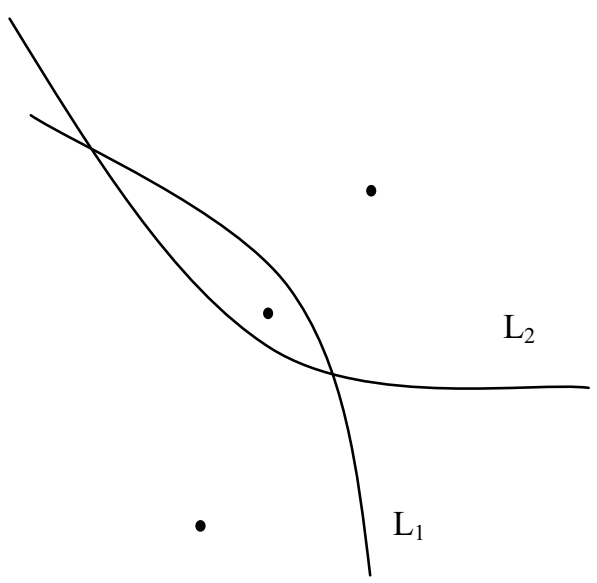

(b)

Figure 1. Schematic diagram of intersection of threestation positioning curves

(a) Positioning curves only with one point of intersection

(b) Positioning curves with two points of intersection

'• 'Receiving station ' $L$ ' Positioning curve

It is relatively complicated to carry out analytical analysis for intersection situation of positioning curves and it is difficult to intuitively see the specific distribution of curve intersection situation if directly in accordance with equation (1), therefore, numerical simulation method is used to conduct analysis for intersection situation of positioning curves. For ideal regular triangle layout of three receiving stations, the distribution situation of intersection point of target positioning curves are as shown in Fig. 2(a). In the figure, white region means that when the target lies in the regional, the positioning curves determined by the target and the stations only has one point of intersection (namely, the target position); shadow area means that when the target lies in the area, the positioning curves determined by the target and the stations have two points of intersection (namely, target position calculation generates ambiguity). For the determined layout of receiving stations, if the target monitored is located at the white area where the positioning curves only have one point of intersection, positioning result selection problem doesn't exist. However, if the target is located at the shadow area where the positioning curves have two points of intersection, the positioning result will generate ambiguity. About how to deal with or avoid such situation, such problem is related to GDOP and stations layout. Thus further analysis will be made as below.

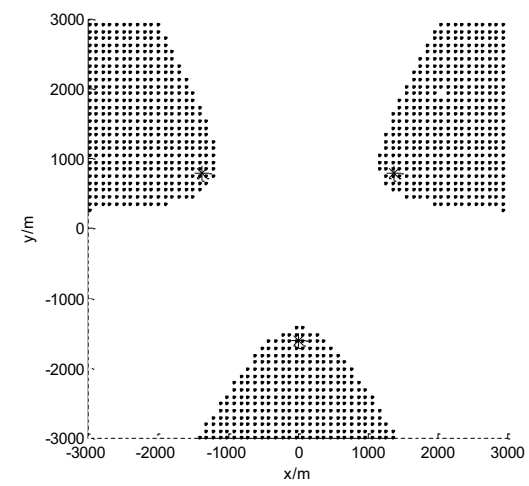

(a)

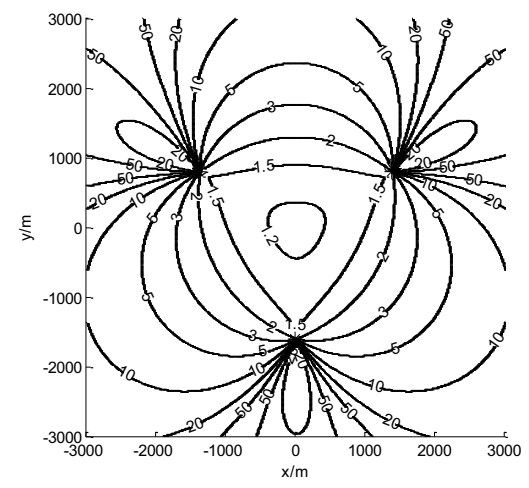

(b)

Figure 2. Intersection point distribution of three-station positioning curve and GDOP distribution (Three stations in the form of regular triangle)

(a) Intersection point distribution of positioning curve

(b) GDOP distribution

'*'Receiving station

\section{Numerical simulation analysis of three-station positioning curve intersection point, GDOP and station layout}

GDOP is a factor establishing relationship between target positioning error and measurement accuracy of time of arrival of the receiving stations realizing target positioning (assuming that measurement error of TOA of target signal of each receiving station realizing target positioning has the same statistics characteristics). GDOP factor describes the influence of the position relationship between multilateration system stations layout and the positioned target on the target positioning accuracy. Assuming that measurement error of TOA of target signal of receiving stations is $\sigma$, target positioning relative standard error in horizontal direction is $\sigma_{x}, \sigma_{y}$ and target positioning relative standard error in vertical direction is $\sigma_{z}$, GDOP is as following [4] : 


$$
G D O P=\sqrt{\sigma_{x}^{2}+\sigma_{y}^{2}+\sigma_{z}^{2}} /(c \cdot \sigma)
$$

Under the situation of two-dimensional positioning, GDOP is:

$$
G D O P=\sqrt{\sigma_{x}^{2}+\sigma_{y}^{2}} /(c \cdot \sigma)
$$

Obviously, for determined TOA measurement accuracy, the smaller GDOP, the smaller corresponding target positioning error is:

$$
G D O P=\sqrt{\sigma_{x}^{2}+\sigma_{y}^{2}+\sigma_{z}^{2}} /(c \cdot \sigma)
$$

GDOP distribution situation of three stations layout shown in Fig. 2(a) is as shown in Fig. 2(b). It can be seen from Fig. 2(b) that central area of triangle formed by three stations is an area with relatively small GDOP, namely, for TOA measurement accuracy of determined receiving station target, target positioning error in such area is relatively small. By comparing Fig. 2(a) with Fig. 2(b), it can be seen that the area where three-station positioning curves have two points of intersection is basically identical with the area with relatively large GDOP, this indicates that the area where three-station positioning curves have two points of intersection not only will generate position calculation error, but also generate relatively large target positioning error even if correct target position can be selected out. In practice, if target TOA measurement accuracy of receiving stations is relatively low, target positioning in such area may lead invalid result due to too large positioning error even.

Fig. 2 is an analysis result under the situation of regular triangle layout of three receiving stations. In order to analyze the influence of stations layout change to positioning curves intersection point distribution and GDOP, the station position can be changed to make qualitative analysis for target positioning curves intersection point distribution and GDOP.

Firstly, move coordinates of one station in Fig. 2 towards inside of the triangle, corresponding target positioning curves intersection point distribution and GDOP are as shown in Fig. 3. It can be seen from Fig. 3 that, with movement of a station towards inside of the triangle, the triangle area surrounded by the stations are reduced and the area where three-station positioning curves have single intersection point and the area with relatively small GDOP are also reduced. However, it can be seen that by comparing Fig. 3(a) and Fig. 3(b), the area where three-station positioning curves have two points of intersection is still basically the area with relatively large GDOP.

Secondly, move coordinates of one station in Fig.2 towards outside of the triangle, corresponding target positioning curves intersection point distribution and GDOP are as shown in Fig. 4. It can be seen from Fig.4 that, with movement of a station towards outside of the triangle, the triangle area surrounded by the stations are increased and the area where three-station positioning curves have single intersection point and the area with relatively small GDOP are also increased. However, it can be seen that by comparing Fig. 4(a) and Fig. 4(b), the area where three-station positioning curves have two points of intersection is still basically the area with relatively large GDOP.

Through the above analysis, the relationship between positioning curves intersection point distribution, GDOP distribution and stations layout at the time of three-station positioning can be qualitatively determined, namely stations layout determines positioning curves intersection point distribution and GDOP, and the area where threestation positioning curves have two points of intersection is basically identical with the area with relative large GDOP. According to the analysis result, when three stations are used for target positioning in practice, please pay attention to select a reasonable stations layout to make the area under monitoring is located at the center of the triangle surrounded by the three stations and the triangle shall "expand" as far as possible, so as to maximize the area where positioning curves have single point of intersection and the area with relatively small GDOP; for the target appearing at the area where the positioning curves have two points of intersection, the positioning result can be given up because it is difficult to judge correct target position and positioning error is relatively large, so as to reduce handling difficulty.

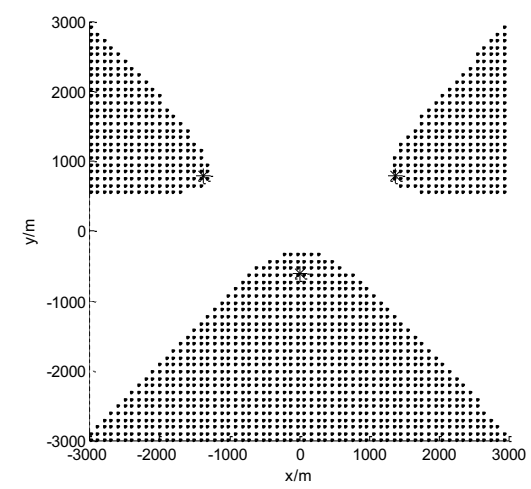

(a)

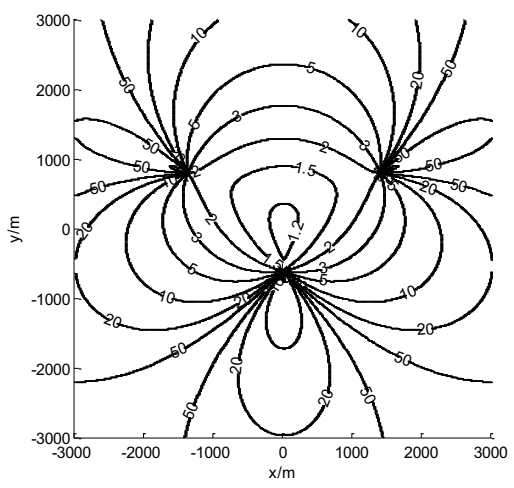

(b)

Figure 3. Three-station positioning curves intersection point distribution and GDOP distribution (one station moves towards inside of the triangle)
(a) Positioning curves intersection point distribution
(b) GDOP distribution
'*'Receiving station 


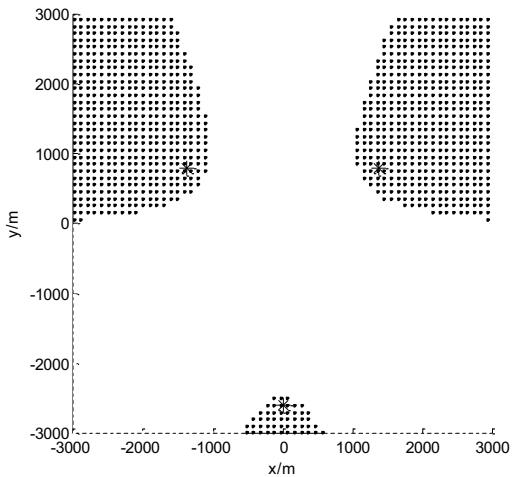

(a)

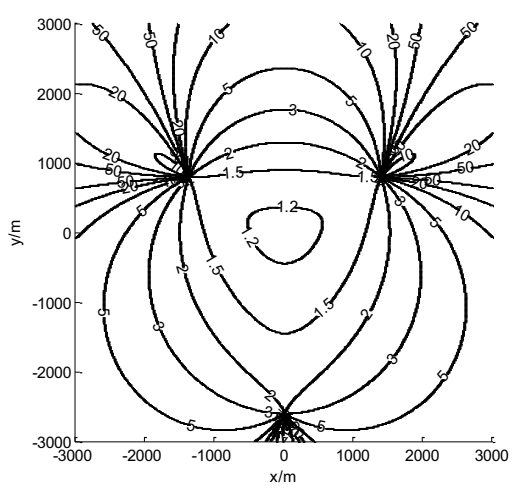

(b)

\section{References}

[1]. International Civil Aviation Organization. ICAO Annex 10 - Aeronautical Telecommunications, Volume IV - Surveillance Radar and Collision Avoidance Systems. International Civil Aviation Organization (2002).

[2]. MH/T4010.Technical standards for ATC secondary surveillance radar (2006).

[3]. Xu zili, Liu Changzhong, He Donglin, et al. Positioning solution using target barometric altitude. Communications Technology (2011).

[4]. H. B. Lee. A novel procedure for assessing the accuracy of hyperbolic multilateration systems. IEEE Trans. Aerospace and Electronic Systems (1975).

Figure 4. Three-station positioning curves intersection point distribution and GDOP distribution (One station moves towards outside of the triangle)
(a) Positioning curves intersection point distribution
(b) GDOP distribution
'*'Receiving station

\section{Conclusions}

The multilateration system will encounter the positioning ambiguity problem if three stations are used for twodimensional target positioning in practical application. The relationship between three-station positioning ambiguity, GDOP and stations layout is numerically analyzed. The qualitative analysis result shows that the distributions of positioning curve intersections and GDOP are results of the stations layout, and the area where the positioning curves have two intersections is basically identical with the area with relative large GDOP. The analysis results can be used as references to avoid and deal with the position calculation ambiguity problem of three-station multilateration positioning target.

\section{Acknowledgment}

This paper is supported by the National Natural Science Foundation of China (Grant No.61079006), and the National Natural Science Foundation of China (Grant No. U1433129). 